\title{
Correction to: Biological and Mechanical Factors Promote the Osteogenesis of Rabbit Artificial Vertebral Laminae: A Comparison Study by Li L, Chen X, He Y, Dong Y. Tissue Eng Part A 2018;24(13-14):1082-1090. DOI: 10.1089/ ten.tea.2017.0426
}

\footnotetext{
N The July 2018 issue of Tissue Engineering, Part A (vol. 24, no. 13-14; 1082-1090) the article entitled "Biological and Mechanical Factors Promote the Osteogenesis of Rabbit Artificial Vertebral Laminae: A Comparison Study” by Li et al. requires correction.
}

A grant in the Acknowledgment section was incorrectly listed. The original Acknowledgment read:

\begin{abstract}
Acknowledgments
“The study was supported by National Natural Science Foundation of China (Grant No. H0606); by Natural Science Foundation of Minhang District, Shanghai (Grant No. 2014MHZ004); and by the Foundation of Shanghai Municipal Commission of Health and Family Planning (Grant Nos. 20134305 and 201440509)."
\end{abstract}

The Acknowledgment should have read:

\section{Acknowledgments}

The study was supported by National Natural Science Foundation of China (Grant No. 81672179); by Natural Science Foundation of Minhang District, Shanghai (Grant No. 2014MHZ004); and by the Foundation of Shanghai Municipal Commission of Health and Family Planning (Grant Nos. 20134305 and 201440509).

The online version of the article has been corrected to reflect this. The authors apologize for this error. 